Biostatistics (2020), 0, 0, pp. 1-11

doi:10.1093/biostatistics/DZR2

\title{
The identity of two meta-analytic likelihoods and the ignorability of double-zero studies
}

\author{
DANKMAR BÖHNING* and PATARAWAN SANGNAWAKIJ \\ Southamption Statistical Sciences Research Institute, University of Southampton \\ Southampton SO17 1BJ, UK \\ and \\ Department of Mathematics and Statistics, Thammasat University \\ Pathum Thani, Thailand \\ d.a.bohning@soton.ac.uk
}

\begin{abstract}
SUMMARY
In meta-analysis, the conventional two-stage approach computes an effect estimate for each study in the first stage and proceeds with the analysis of effect estimates in the second stage. For counts of events as outcome, the risk ratio is often the effect measure of choice. However, if the metaanalysis includes many studies with no events the conventional method breaks down. As an alternative one-stage approach a Poisson regression model and a conditional binomial model can be considered where no event studies do not cause problems. The conditional binomial model excludes double-zero studies, whereas this is seemingly not the case for the Poisson regression approach. However, we show here that both models lead to the same likelihood inference and double-zero studies (in contrast to single-zero studies) do not contribute in either case to the likelihood.
\end{abstract}

Key words: Conditional binomial likelihood; data fusion; double-zero studies; Poisson likelihood; meta-

*To whom correspondence should be addressed. 
analysis

\section{INTRODUCTION}

Meta-analysis and data fusion of studies with rare events has become recently a matter of prime interest. An example is the post-admission surveillance of the anti-diabetes drug Rosiglitazone where concern arose in terms of adverse events. A meta-analysis of Nissen \& Wolski $(2007,2010)$ showed an increased risk ratio for myocardial infarction while being on treatment. This metaanalysis started a debate on how to deal with rare event studies in a meta-analysis. For a review on this debate see Böhning et al. (2015). The central problem is the occurrence of single-zero and double-zero trials, former being a trial where exactly in one arm a zero count occurs, the latter being a trial where both arms contain zero counts as study outcome. To demonstrate the problem we look at a meta-analysis on perinatal death in post-term pregnancy comparing selective induction with routine induction. The data are taken from Piaget-Rossel \& Taffé (2019a) (originally stemming from Crowley (2010)) and provided in Table 1 for convenience. The metaanalysis consists only of zero-studies, eleven double-zero studies and eight single-zero studies. Note that the studies are not small in arm size, the smallest having ten at risk while the largest has 1706 at risk. It is clear that a conventional two-stage meta-analysis is not possible for these kind of data. Recall that a two-stage meta-analysis proceeds, in principle, as follows. In the first stage an effect measure such as the risk ratio is determined for each study which generates a sample of study statistics which are in a second stage further analyzed by computing a summary statistic, for example, and performing heterogeneity analysis. For a review of the approach see, for example, the recent works by Borenstein et al. (2009) or Schwarzer et al. (2015). The two-stage approach fails for the data of Table 1 as for none of the nineteen studies the risk ratio can be reliably estimated. For a discussion and review of rare event meta-analysis including zero-counts see also Lane (2013) and for an alternative approach based on the arcsine-transformation which 
Table 1. Meta-analytic data set of 19 studies on perinatal death in post-term pregnancy comparing selective with routine induction

\begin{tabular}{lcrcr} 
& \multicolumn{2}{c}{ Selective induction } & \multicolumn{2}{c}{ Routine induction } \\
Study $i$ & Deaths & At risk & Deaths & At risk \\
1 & 0 & 55 & 2 & 57 \\
2 & 0 & 118 & 0 & 119 \\
3 & 0 & 131 & 1 & 134 \\
4 & 0 & 57 & 0 & 55 \\
5 & 0 & 481 & 0 & 235 \\
6 & 1 & 78 & 0 & 78 \\
7 & 0 & 66 & 0 & 53 \\
8 & 0 & 76 & 0 & 90 \\
9 & 0 & 195 & 1 & 207 \\
10 & 0 & 214 & 0 & 195 \\
11 & 0 & 152 & 1 & 150 \\
12 & 0 & 103 & 0 & 97 \\
13 & 0 & 94 & 1 & 94 \\
14 & 0 & 188 & 1 & 168 \\
15 & 0 & 12 & 0 & 10 \\
16 & 0 & 109 & 0 & 129 \\
17 & 0 & 1701 & 2 & 1706 \\
18 & 0 & 57 & 0 & 51 \\
19 & 0 & 235 & 0 & 175
\end{tabular}

can cope with zero-event studies as well see Rücker et al. (2009). Other recent contributions include the works of Piaget-Rossel \& Taffé (2019a) and Piaget-Rossel \& Taffé (2019b).

We consider the one-stage approach based upon generalized linear models. It appears reasonable to use a Poisson regression model for the data of Table 1, treating the count of deaths as Poisson outcome, conditional on a linear predictor which includes a binary covariate for the type of induction, a fixed effect for each study as well as the log-number at risk as offset. This approach has been suggested, for example, in Cai et al. (2010).

Yet, another approach (also mentioned in Cai et al. (2010), Stijnen at al. (2010) or Böhning et al. (2015)) considers the fact that the count in the intervention conditional upon the sum of both outcome over the study arms is binomial with the sum of both count outcomes as size parameter and the event parameter only involving the parameter of interest. This is an attractive 
approach as it does not involve a main effect for each study any more, hence eliminates the baseline parameter and only involves the parameter of interest, $R R$. It can be seen as moving from a two-sample problem to an equivalent one-sample problem. However, it is often argued against this approach that double-zero studies do not play any role as for those the binomial denominator is zero, whereas it is felt that they contribute in the Poisson regression approach mentioned in the previous paragraph (see also Kuss (2015) for this aspect). See also Piaget-Rossel \& Taffé (2019c) for an investigation of the role of zero-event studies in rare-events meta-analysis.

We have implemented both approaches, the Poisson regression and the conditional, binomial model for the data of Table 1 and present the results in Table 2. We find the result, potentially surprising, that the inference based upon the Poisson regression model and the conditional, binomial coincides entirely. Hence also double-zero studies do not contribute to the Poisson regression model, potentially, in contrast to common belief.

Table 2. One-stage estimates of the risk ratio for the meta-analytic data set of 19 studies on perinatal death in post-term pregnancy comparing selective with routine induction (Table 1); the main effects for the Poisson regression model are not given here for brevity; note also that the conditional binomial approach requires no other estimate than the intercept given here

$\begin{array}{lccccc}\text { Model } & \text { Risk ratio } & \text { SE } & z & \text { P-value } & \text { CI } \\ \text { Poisson regression } & 0.1114 & 0.1174 & -2.08 & 0.037 & 0.0141-0.8795 \\ \text { conditional binomial } & 0.1114 & 0.1174 & -2.08 & 0.037 & 0.0141-0.8795 \\ \text { Mantel-Haenszel } & 0.1113 & & & & 0.0141-0.8799\end{array}$

This short note is organized as follows. In section 2 we introduce notations and models in the context of a single study. In section 3 we discuss the general meta-analytic setting and show the identity of the log-likelihoods for the Poisson regression model with the conditional, binomial model. Section 4 closes with a short discussion including the connection to Mantel-Haenszel estimation. 


\section{A Poisson LIKELIHOOd}

For simplicity, we start with the situation of one study. To be slightly more general, we allow units to be different with respect to their times under risk and denote with $T_{0}$ and $T_{1}$ the persontimes in control and intervention arm. If all units are under risk for the same time period, the person-time reduces to the number of units under risk as this is the case for the meta-analytic data presented in Table 1. Let $X_{0}$ denote a Poisson count for a control arm with mean $\mu_{0}>0$ and person-time $T_{0}$ so that

$$
\log E\left(X_{0}\right)=\log \mu_{0}=\log T_{0}+\alpha
$$

with $\alpha$ being a real number. Also, let $X_{1}$ denote a Poisson count for an intervention arm with mean $\mu_{1}>0$ and person-time $T_{1}$ so that

$$
\log E\left(X_{1}\right)=\log \mu_{1}=\log T_{1}+\alpha+\beta
$$

with $\beta$ being also a real numbers. In fact, $e^{\beta}$ denotes the risk ratio

$$
R R=e^{\beta}=\frac{\mu_{1} / T_{1}}{\mu_{0} / T_{0}}
$$

It is assumed that $X_{1}$ and $X_{0}$ are independent. We also emphasize that, from our perspective, the Poisson assumption in the rare event situation is reasonable, at least a wide-spread assumption. For an approach utilizing the negative-binomial distribution see Piaget-Rossel \& Taffé (2019b).

For a single study, the associated log-likelihood corresponds up to an additive constant independent of $\alpha$ and $\beta$

$$
\begin{aligned}
& \log L=-T_{1} e^{\alpha} e^{\beta}+x_{1}\left(\log T_{1}+\alpha+\beta\right)-T_{0} e^{\alpha}+x_{0}\left(\log T_{0}+\alpha\right) \\
& =-e^{\alpha}\left(T_{0}+T_{1} e^{\beta}\right)+x \alpha+x_{1} \beta=-e^{\alpha} T_{0}\left(1+r e^{\beta}\right)+x \alpha+x_{1} \beta
\end{aligned}
$$

where the last equality is valid up to an additive constant again, $x=x_{0}+x_{1}$ and $r=T_{1} / T_{0}$. Keeping $\beta$ fixed, (2.3) is maximised for $e^{\hat{\alpha}}=x /\left[T_{0}\left(1+r e^{\beta}\right)\right]$ and the resulting profile log-likelihood 
function is given as

$$
\ell(\beta)=x_{1} \beta-x \log \left(1+r e^{\beta}\right)
$$

again up to some additive constant independent of $\beta$.

\section{A COnditional Likelihood}

We refer to a well-known result (Ross, 1985) that $X_{1}$ conditional upon $X_{1}+X_{0}=x$ is binomial with size parameter $x$ and event parameter $q=\frac{\mu_{1}}{\mu_{1}+\mu_{0}}$ :

$$
P\left(X_{1}=x_{1} \mid X_{1}+X_{0}=x\right)=\left(\begin{array}{c}
x \\
x_{1}
\end{array}\right) q^{x_{1}}(1-q)^{x_{0}},
$$

using the fact that $X_{1}$ and $X_{0}$ are independent Poisson counts with parameters $\mu_{1}$ and $\mu_{0}$, respectively. The binomial likelihood (3.5) has the benefit that the baseline parameter $\alpha$ is eliminated. This can be seen by considering

$$
q=\frac{\mu_{1}}{\mu_{1}+\mu_{0}}=\frac{T_{1} e^{\alpha} e^{\beta}}{T_{1} e^{\alpha} e^{\beta}+T_{0} e^{\alpha}}=\frac{r e^{\beta}}{1+r e^{\beta}}=\frac{r R R}{1+r R R}
$$

Hence the main effect of the factor study drops out. In the case of balanced studies $\left(T_{1}=T_{0}\right.$ or $r=1)$ the binomial event parameter $q$ is simply $R R /(1+R R)$ where $R R$ is the risk ratio.

The associated log-likelihood function (up to an additive constant independent of $\beta$ ) is

$$
\ell(\beta)=x_{1} \beta-x \log \left(1+r e^{\beta}\right)
$$

which is evidently identical to (2.4). Both likelihoods show that a double-zero study (in contrast to a single-zero study) does not provide any information as their associated log-likelihood is zero.

\section{MAJOR RESULT}

We now consider the more general meta-analytic setting of $k$ studies with independent Poisson counts $X_{i j}$ for study $i=1,2, \cdots, k$ and arm $j=0,1$. The notation $j=1$ identifies the treatment 
arm and $j=0$ the control arm. We assume the model

$$
\log E\left(X_{i j}\right)=\log T_{i j}+\alpha_{i}+\beta \times j
$$

where $T_{i j}$ is the person-time in study $i$ and $\operatorname{arm} j, i=1,2, \cdots, k$ and $j=0,1$. Note that model (4.7) has study-specific intercepts but a common risk ratio $e^{\beta}$ across studies. It is important to include study-specific intercept terms as one would like to retain the within-study intervention comparison. The log-likelihood is then (up to an additive constant)

$$
\log L=-\sum_{i} e^{\alpha_{i}} T_{i 0}\left(1+r_{i} e^{\beta}\right)+\sum_{i} x_{i} \alpha_{i}+\sum_{i} x_{i 1} \beta
$$

where $x_{i}=x_{i 0}+x_{i 1}$ and $r_{i}=T_{i 1} / T_{i 0}$. The log-likelihood (4.8) is maximized for fixed $\beta$ by $e^{\hat{\alpha}_{i}}=x_{i} /\left[T_{i 0}\left(1+r_{i} e^{\beta}\right)\right]$. We achieve the profile log-likelihood function

$$
\ell(\beta)=\sum_{i} x_{i 1} \beta-\sum_{i} x_{i} \log \left(1+r_{i} e^{\beta}\right)
$$

The conditional binomial likelihood generalizes for $k$ studies to

$$
\prod_{i}\left(\begin{array}{c}
x_{i} \\
x_{i 1}
\end{array}\right) q_{i}^{x_{i 1}}\left(1-q_{i}\right)^{x_{i 0}}
$$

where $q_{i}=\frac{r_{i} e^{\beta}}{1+r_{i} e^{\beta}}$. The $\log$-likelihood associated with (4.10) is identical to (4.9) up to an additive constant independent of $\beta$. Note that

$$
\operatorname{logit}\left(q_{i}\right)=\log q_{i}-\log \left(1-q_{i}\right)=\log r_{i}+\beta,
$$

so that (4.10) is easily maximized by means of a logistic regression model with event count $x_{i 1}$ and binomial denominator $x_{i}=x_{i 0}+x_{i 1}$ as dependent variable and linear predictor $\log r_{i}+\beta$, the latter being only an intercept model with offset term $\log r_{i}$, the $\log$-ratios of the persontimes of intervention and control arm in study $i$. Note that the intercept term in (4.11) is still the original log-risk ratio, in contrast to the usual interpretation in logistic regression. Table 3 presents the data in a way that they can be readily analyzed using a logistic regression fit with 
Table 3. Conditional, binomial representation of meta-analytic data set of 19 studies on perinatal death in post-term pregnancy comparing selective with routine induction

$\begin{array}{lccc}\text { Study } i & x_{1 i} & x_{i} & r_{i} \\ 1 & 0 & 2 & 0.97 \\ 2 & 0 & 0 & 0.99 \\ 3 & 0 & 1 & 0.98 \\ 4 & 0 & 0 & 1.04 \\ 5 & 0 & 0 & 2.05 \\ 6 & 1 & 1 & 1.00 \\ 7 & 0 & 0 & 1.25 \\ 8 & 0 & 0 & 0.84 \\ 9 & 0 & 1 & 0.94 \\ 10 & 0 & 0 & 1.10 \\ 11 & 0 & 1 & 1.01 \\ 12 & 0 & 0 & 1.06 \\ 13 & 0 & 1 & 1.00 \\ 14 & 0 & 1 & 1.12 \\ 15 & 0 & 0 & 1.20 \\ 16 & 0 & 0 & 0.85 \\ 17 & 0 & 2 & 1.00 \\ 18 & 0 & 0 & 1.12 \\ 19 & 0 & 0 & 1.34\end{array}$

only an intercept term, the log-relative risk, and the log-ratio of the person-times. Finally, we can think of the conditional, binomial likelihood as a profile likelihood for a Poisson regression model where the main effect of study has been eliminated by means of profiling.

\section{Discussion}

We close with a few remarks. The suggested approach has a close connection to Mantel-Haenszel estimation which is popular in epidemiology. Consider the score w.r.t. (4.9) which is given as

$$
\sum_{i} x_{i 1}-\sum_{i} x_{i} \frac{r_{i} R R}{1+r_{i} R R}=\sum_{i} \frac{x_{i 1} T_{i 0}}{T_{i 0}+T_{i 1} R R}-\sum_{i} \frac{x_{i 0} T_{i 1}}{T_{i 0}+T_{i 1} R R} R R .
$$

Equating this score to zero leads to

$$
R R=\frac{\sum_{i} T_{i 0} x_{i 1} /\left(T_{i 0}+T_{i 1} R R\right)}{\sum_{i} T_{i 1} x_{i 0} /\left(T_{i 0}+T_{i 1} R R\right)}
$$


Equation (5.12) cannot only be seen as an implicit characterization of the maximum likelihood estimator, it also provides an iterative scheme for generating it by starting with some value for $R R$ such as $R R=1$, generating a new value by computing the right-hand side of (5.12), plugging that in and so forth. The first iterate when starting with $R R=1$ is the Mantel-Haenszel estimate $\sum_{i} w_{i} T_{i 0} x_{i 1} / \sum_{i} w_{i} T_{i 1} x_{i 0}$, where $w_{i}=1 /\left(T_{i 0}+T_{i 1}\right)$. Note that maximum likelihood and MantelHaenszel estimator coincide if all studies are balanced $\left(r_{i}=1\right)$. This explains why for the data of Table 1 both estimators are very close as the studies are nearly balanced (see also Tables 2 and 3). Note that the Mantel-Haenszel estimator is always defined (unless one arm has only zeros in all studies) and is also invariant to the inclusion or exclusion of double-zero studies (whereas it can change its value when single-zero studies are excluded as pointed out in Böhning et al. (2015)). Mantel-Haenszel estimation is popular but has limitations when it comes to the inclusion of further covariates. The latter would be easily possible for the conditional logistic regression model by extending the linear predictor by further covariates such a time of place of study, intervention modification or size of study.

The conditional binomial can also easily be extended to include a parametric random effect for the intervention as follows

$$
\prod_{i}\left(\begin{array}{c}
x_{i} \\
x_{i 1}
\end{array}\right) \int_{\beta_{i}}\left(\frac{r_{i} e^{\beta_{i}}}{1+r_{i} e^{\beta_{i}}}\right)^{x_{i 1}}\left(\frac{1}{1+r_{i} e^{\beta_{i}}}\right)^{x_{i 0}} \phi\left(\beta_{i} \mid \beta_{0}, \tau^{2}\right) d \beta_{i},
$$

where $\phi\left(\beta_{i} \mid \beta_{0}, \tau^{2}\right)$ could be the normal density with mean $\beta_{0}$ and variance $\tau^{2}$. This would allow investigating for heterogeneity of the intervention effect across studies. This is clearly simpler than using the Poisson regression model where two nested marginals have to be considered.

\section{SOFTWARE AND DATA}

We have used the package STATA (version 16) for the analysis of the meta-analytic data in this paper. Code and data can be found at https://github.com/boehning/meta_likelihood. The provided code will reproduce all analysis in this paper and can also be used for similar meta- 
analytic event data and analysis.

\section{REFERENCES}

Böhning, D., Mylona, K. \& Kimber, A. (2015). Meta-analysis of clinical trials with rare events. Biometrical Journal $57633-648$.

Borenstein, M., Hedges, L. V., Higgins, J. P. \& Rothstein, H. R. (2009). Introduction to Meta-Analysis. Chichester: John Wiley \& Sons.

Cai, T., Parast, L. \& Ryan, L. (2010). Meta-analysis for rare events. Statistics in Medicine $292078-2089$.

Crowley, P. (2000). Interventions for preventing or improving the outcome of delivery at or beyond term. Cochrane Database of Systematic Reviews 2 CD000170.

Kuss, O. (2015). Statistical methods for meta-analyses including information from studies without any eventsadd nothing to nothing and succeed nevertheless. Statistics in Medicine $\mathbf{3 4}$ 1097-1116.

Lane, P.W. (2013). Meta-analysis of incidence of rare events. Statistical Methods in Medical Research 22 117-132.

Nissen, S. E. \& Wolski, K. (2007). Effect of Rosiglitazone on the risk of myocardial infarction and death from cardiovascular causes. N. Engl. J. Med. 356 2457-2471.

Nissen, S. E. \& Wolski, K. (2010). Rosiglitazone revisited: an updated meta-analysis of risk for myorcial infarction and cardiovascular mortality. Arch. Intern. Med. 170 1191-1201.

Piaget-Rossel, R. \& Taffé, P. (2019a). Meta-analysis of rare events under the assumption of a homogeneous treatment effect. Biometrical Journal 61 1557-1574. 
Piaget-Rossel, R. \& Taffé, P. (2019b). Meta-analysis of incidence rate data in the presence of zeroevent and single-arm studies. International Journal of Statistics in Medical Research $\mathbf{8}$ $57-66$.

Piaget-Rossel, R. \& TAffé, P. (2019c). A pseudo-likelihood approach for the meta-analysis of homogeneous treatment effect: exploiting the information contained in double-zero and singlearm studies. Journal of Statistics: Advances in Theory and Applications 21 91-117.

Ross, S. M. (1985). Introduction to Probability Models. Oxford: Elsevier Inc.

Rücker, G., Schwarzer, G., Carpenter, J. \& Olkin, I. (2009). Why add anything to nothing? The arcsine difference as a measure of treatment effect in meta-analysis with zero cells. Statistics in Medicine 28 721-738.

Schwarzer, G., Carpenter, J. \& RüCKer, G. (2015). Meta-Analysis with R. Heidelberg: Springer.

Stijnen, T., Hamza, T.H. \& Ozdemir, P. (2010). Random effects meta-analysis of event outcome in the framework of the generalized linear mixed model with applications in sparse data. Statistics in Medicine 29 3046-3067.

[Received August 1, 2019; revised October 1, 2019; accepted for publication November 1, 2019] 